\title{
When brain rhythms aren't "rhythmic": implication for their mechanisms and meaning
}

\section{Stephanie R. Jones}

\begin{abstract}
Rhythms are a prominent signature of brain activity. Their expression is correlated with numerous examples of healthy information processing and their fluctuations are a marker of disease states. Yet, their causal or epiphenomenal role in brain function is still highly debated. We review recent studies showing brain rhythms are not always "rhythmic", by which we mean representative of repeated cycles of activity. Rather, high power and continuous rhythms in averaged signals can represent brief transient events on single trials whose density accumulates in the average. We also review evidence showing time-domain signals with vastly different waveforms can exhibit identical spectral-domain frequency and power. Further, nonoscillatory waveform feature can create spurious high spectral power. Knowledge of these possibilities is essential when interpreting rhythms and is easily missed without considering preprocessed data. Lastly, we discuss how these finding suggest new directions to pursue in our quest to discover the mechanism and meaning of brain rhythms.
\end{abstract}

\section{Introduction}

Oscillations in electrical signatures of neural activity occur in almost all structures in the brain across electrophysiological scales ranging from rhythmic activities in single neurons to largescale population dynamics as measured with magneto- and electro-encephalography (MEG/EEG). Modulations in rhythms correlate with behaviors such as perception, attention, memory and motor action [1-4, 5*, 6-9]. Their disruption is a biomarker of several disease states, most notably Parkinson's Disease [10], autism [11] and schizophrenia [12]. Causal manipulations of brain rhythms with techniques such as ontogenetic [13], transcranial alternating current stimulation (tACS)[14], and repetitive transcranial magnetic stimulation (rTMS) [15] have shown that oscillatory entrainment can actively modulate behavior. While studies connecting rhythms to function are vast and rapidly growing, our understanding of their causal and/or epiphenomenal role in information processing is still highly debated. Some view rhythms as essential to temporally coordinating activity necessary for information processing [16-20], while others suggests rhythms are an epiphenomenal reflection of other key processes [21-25]. 
Essential to defining the role of rhythms in function is an accurate interpretation of the cellular and network level mechanisms underlying the neurophysiological data. In our view, this interpretation relies on a detailed understanding of the nature of the rhythm in the un-averaged, unfiltered time-domain signal. Typically, many levels of filtering and averaging are applied to time series data before a connection between rhythms and function is made, and it is uncommon for raw data to be shown in any form. While such analyses are often necessary to limit the scope of question, and/or show statistical significance of results, we argue here that these practices can lead to misinterpretations in tying a frequency band of activity to a particular mechanism or hypothesized function.

We review several lines of evidence investigating unaveraged data that demonstrate brain activity that shows functionally relevant changes in the power spectrum of averaged data is not always "rhythmic", defined as exhibiting repeated cycles of activity with a reliable period. First, we review recent studies that reveal rhythmic activity on individual trials is often transient [26*, $\left.27^{\star \star}, 28^{\star \star}, 29^{\star \star}\right]$. The accumulation of these transient activations across trials results in a prolonged high-power oscillation in the average, creating the illusion of a sustained rhythm. As such, differences in averaged power across behavioral conditions can reflect a change in the accumulation of transients across trials rather than a change in the net amplitude or duration of the oscillations $\left[28^{* \star}, 29^{* *}\right]$.

Second, we review evidence demonstrating that peaks at specific frequencies in the spectrum can be created by dominant waveform features in the time domain rather than periodic "sinusoidal" type oscillations. While the waveform may retain oscillatory components, peaks in the spectral domain can also emerge as a spurious consequence of specific waveform shapes $\left[30^{\star}, 31^{\star \star}, 32^{\star \star}, 33^{\star \star}\right]$. Lastly, we discuss how these findings imply a need to develop a new generation of analysis methods to study rhythms and suggest several techniques that may help uncover their meaning for function.

\section{Sustained and high spectral power in the average can reflect the accumulation of transient "rhythmic" events across trials}


When we think of rhythms, we typically imagine repeated cycles of oscillatory activity. Indeed, there are many instances of rhythms in the brain where this is the case, including the wellknown examples of eyes-closed $(7-14 \mathrm{~Hz})$ alpha rhythms over occipital cortex [34], sleep rhythms [35] and hippocampal $(4-8 \mathrm{~Hz})$ theta rhythms $[17,20,36]$. However, recent evidence shows that in many cases brain signals considered as belonging to a frequency-defined class of brain rhythms do not represent sustained oscillation but rather brief bouts of activity that are repeated intermittently $\left[26^{\star}, 28^{\star \star}, 29^{\star *}\right]$, see also $[37,38]$. This intermittency has a direct impact on the interpretation of the meaning of an oscillation, as the potential influence can be quite different if a rhythm is sustained for many cycles versus only one or two.

The often false notion that brain rhythms are sustained comes from the standard procedure of averaging in the spectral domain $\left[39^{\star *}\right]$, see also $\left[40,41^{\star}\right]$. When frequency analysis is applied to a time series signal, the power representation of the signal across time is purely nonnegative. Thus, when averaging across trials in the spectral domain, transient bouts of positive spectral power accumulate without cancellation (Figure 1A; e.g. induced rhythms). In contrast, when averaging across trials in the temporal domain, positive and negative signals cancel resulting in low power in the spectrogram of the average (Figure 1B). In the latter case, rhythms emerge in the averaged signal only when they are time locked across trials (e.g. evoked rhythms) or if their amplitude on a single trial is sufficiently large to persist in the average. The non-zero accumulation in the spectral domain (Figure 1A) has two implications: (1) rhythms can appear as prolonged in duration in the average, and (2) high power activity at a point in time in the average can reflect repeatability in the timing of the brief bout of activity across individual trials rather than an increase in "amplitude" of the signal. The latter could also be reflected as inter-trial phase coherence [42,43], which some studies have associated with the resetting of ongoing oscillations [44-46]. However, phase consistency across trials is not necessary to observe high power in the average.

By investigating the nature of unaveraged brain signals, several recent studies have shown transient "rhythms" exist in different brain areas and species. Figure 1 depicts examples of spontaneous (pre-stimulus) low frequency alpha and beta $(15-29 \mathrm{~Hz})$ rhythms emerging as part of the so called Rolandic mu-complex from human MEG data source localized to primary somatosensory cortex (SI) (adapted from $\left[26^{\star}, 27^{\star *}\right]$ ). In this data, we showed that on individual trials, bouts of high power in the alpha and beta bands emerge transiently and intermittently, 
typically lasting $<150 \mathrm{~ms}\left[26^{\star}, 27^{\star *}\right]$. However, in the average across 100 trials the rhythms appear as continuous bands of activity. This effect is also reflected as peaks in the average power spectral density (see Figure 3 in [26*]).

The transient nature of rhythms has implications for their role in function. Beta band activity in local field potential (LFP) recordings in motor cortex and striatum from awake behaving monkeys has also been shown to be transient on individual trials (typically $<150 \mathrm{~ms}$ ), coined as beta "bursts" [28**]. In these signals, changes in averaged power across trials and conditions were shown to reflect modulation of "burst" probabilities. Burst densities peaked at different times in the different regions reflecting differences in motor and cognitive demands during the task. Most recently, working memory has been shown to be associated with transient emergence of beta and gamma $(45-100 \mathrm{~Hz})$ activity in prefrontal cortex [29**] (beta burst duration $=130 \mathrm{~ms}+/-37 \mathrm{~ms}$; gamma burst duration $67+/-19 \mathrm{~ms}$ ). Beta bursts were infrequent during encoding and decoding, whereas firing in neurons predictive of these processes correlated with changes in gamma burst rates. Gamma burst rates also increased as memory load increased, which the authors suggested was reflective of the number of spiking cell assemblies becoming active.

\section{Dominant waveform features can create rhythms that do not represent repeated bouts of activity with direct implications on their mechanism}

Here, we review evidence suggesting features of the raw time-domain signal are also essential to consider when interpreting the mechanisms or meaning of a rhythm. Vastly different waveform shapes, created by different underlying mechanisms, can create identical peaks in spectral power and/or spurious high power activity.

\section{Spectral peaks created by sinusoidal vs. non-sinusoidal waveform features}

A common procedure in the study of neural rhythms is to band-pass the time domain signal into frequencies of interest, often in a narrowband window. By construction, band-pass techniques force a sinusoidal shaped waveform of varying amplitude onto the signal. This process along with de-trending (e.g. mean subtracting) the data can give the sense that the underlying waveforms oscillate symmetrically around some mean value. However, non-sinusoidal signals 
can produce peaks in power spectra at the same frequency and power as a sinusoidal signal, and the neural mechanisms underlying these different signatures of the same "rhythm" can be fundamentally different.

Figure 2 shows three example signals with vastly different waveforms that create transient peaks in time-frequency spectral power at an identical beta frequency $(22 \mathrm{~Hz})$ and with approximately equal duration ( $150 \mathrm{~ms})$. The signals represent: 3 cycles of a $22 \mathrm{~Hz}$ sinusoidal rhythm (Figure 2A), an inverted Ricker wavelet with a dominant peak that last 40ms (Figure 2B), and a single 33ms deflection that does not oscillate a full cycle (Figure $2 \mathrm{C}$ ). Knowledge of these time-domain differences can be crucial to identifying the neural generator of the rhythm.

For example, several modeling and experimental studies, primarily from slice recordings, have established that beta frequency rhythms consisting of several cycles with a regular period (e.g. Figure $2 \mathrm{~A}$ ) can emerge in local neocortical circuits via the spiking interactions of excitatory and inhibitory neurons [47**-49]. In these studies, beta frequency activity in the LFP coincided with repeated bouts of pyramidal neuron firing with an inter-"burst "interval in the beta band, here $45 \mathrm{~ms}, 22 \mathrm{~Hz}$ (see schematic spiking in Figure $2 \mathrm{~A}$; the term burst here is used to mean one or more spikes across the population).

In contrast, we recently showed that transient beta events in source localized MEG signals from $\mathrm{SI}$ had a stereotypical waveform shape reminiscent of Figure 2B (e.g. see red boxes in Figure 1B) $\left[27^{\star \star}\right]$. Computational neural modeling designed to accurately reflect the biophysics of human current source signals $\left[26^{\star}, 27^{\star \star}, 30^{\star}, 50,51^{\star}\right]$, combined with in vivo laminar LFP recordings in animal models, suggested that the dominant peak defining the high-power beta event emerged from a strong burst of synaptic drive to pyramidal neuron distal dendrites that lasted approximately one beta period (see burst schematic in Figure 2B) [27**]. Thus, evidence indicates that there are at least two different mechanisms for beta that are reflected in different characteristic waveforms. While the spectrograms reflective of these processes show qualitative differences (Figure 2 top panels), the potential to disambiguate between these possible mechanisms is lost without considering the raw waveforms and accurately accounting for the biophysics of the signal. 


\section{High frequency rhythms and spurious cross-frequency coupling created by fast transitions in waveforms}

A consistent theme in the study of neural oscillations is the amplitude coupling of a high frequency rhythm to the phase of a lower-frequency oscillation. Most often, high- $(>80 \mathrm{~Hz})$ or low $(30-60 \mathrm{~Hz})$ gamma rhythms are shown to be embedded within slower frequency oscillations, such as theta or alpha, and such coupling has been correlated with information processing on a variety of time-scales $[17,20,52,53]$. Cross-frequency coupling can occur because of two underlying neural oscillations interacting in meaningful way, but it can also be a spurious consequence of specific waveform features that are not representative of an independent neural processes. Because of their hypothesized roles in information processing, the number of studies investigating cross frequency coupling is growing rapidly. As such, it is timely to review the fact that there are several ways spurious cross-frequency coupling can occur, particularly in the high-gamma band.

Kramer, Tort and Kopell (2008) succinctly showed that spectral analysis techniques applied to slow oscillations that include fast transitions (i.e. sharp edges) create coupling between the amplitude of gamma frequency oscillations and the phase of underlying slow oscillation [31**]. This coupling comes from the fact that when spectral analysis techniques (e.g. multitaper methods, short time Fourier transforms, matching pursuit, etc., see [54-56*] for reviews of spectral methods) are applied to sharp transitions they produce broadband power increases, including increases through the high-gamma band. Figure $3 \mathrm{~A}$ illustrates this fact with an extreme example of a sawtooth waveform that exhibits sharp transitions between cycles. Standard spectral analysis applied to this signal (7 cycle Morlet wavelet convolution) shows bands of high power activity not only at the frequency of the sawtooth $(22 \mathrm{~Hz})$, but at twice the frequency $(44 \mathrm{~Hz})$, and across a broad band of high gamma power $(>60 \mathrm{~Hz})$. The amplitude of the high gamma power is spuriously coupled to the phase of the underlying $22 \mathrm{~Hz}$ oscillation during the falling phases of each sharp edge. Such spurious coupling occurs even when the transitions in the waveform are more modest (see Figure 2 in [31**]).

Based on the same principle, Ray and Maunsell (2011) have shown that high-band gamma activity $(>80 \mathrm{~Hz}$ ) in LFP signals from primary visual cortex in monkeys is related to population spiking, measured as multi-unit activity and not to be confused with isolated neurons firing at a 


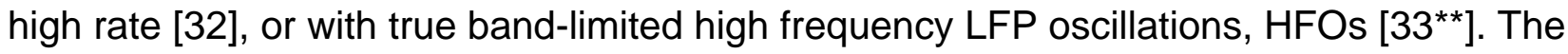
authors note that their results rely on the fact that spikes produce sharp transients in the LFP that have a broadband signature. At lower frequencies $<\sim 50 \mathrm{~Hz}$ the broadband activity is masked by $1 / f$ noise in their data. The fact that high-gamma activity can be created by sharp waveform transitions is well-known to those who have studied spectral analysis methods. A large majority of studies, however, do not take this possibility into consideration, and without investigating unaveraged data this prospect is easily missed.

Coupling between high-gamma and lower frequency oscillations can also emerge from fast oscillatory waveform features that do not represent broadband sharp edge effects or population spiking. Lee and Jones (2013) employed a biophysically principled model of human current dipole signals to investigate dipole waveform features that could distinguish between alternate network mechanisms creating gamma band oscillations [30*]. We showed that low frequency $(\sim 50 \mathrm{~Hz})$ gamma oscillations simulated with well-established pyramidal-interneuron spiking interactions (i.e. PING mechanism $\left[47^{* *}, 57,58\right]$ ) creates single cycles of a fast high-gamma oscillation $(-110 \mathrm{~Hz})$ in the dipole waveform and hence coupling between periods of high and low gamma power (Figure 3B). Figure 3Bi depicts the dipole waveform simulated with the PING mechanism where the oscillation starts with firing in the pyramidal population (black raster and histogram in Bii), followed by synaptically induced firing in the inhibitory neurons (red raster) that keeps the pyramidal neurons from firing for $\sim 20 \mathrm{~ms}$ (the period of a low frequency gamma rhythm). This mechanism creates clear low gamma power in the spectrogram, where peaks in low gamma $(\sim 5 \mathrm{OHz})$ occur at the time as peaks in high gamma $(\sim 110 \mathrm{~Hz})$ power (Figure 3Bi; red box highlights a window of time-locked coupling of low and high gamma power). A closer inspection of the dipole waveform during each bout of pyramidal-interneuron spiking shows a high frequency $\sim 110 \mathrm{~Hz}$ gamma oscillation (see red dotted line on dipole waveform in Figure 3Bii middle-bottom panel; time window corresponds to red box in Figure 3Bi) that is also reflected in the corresponding spectrogram (Figure 3Bii, bottom panel). It is important to emphasize that the temporal "coupling" of low and high gamma power in the spectrogram was created by one underlying mechanism, namely a single bout of pyramidal-interneuron spiking that created both low and high frequency gamma oscillations in the current dipole waveform. In contrast, the interpretation of the origin of high-gamma activity could easily be misconstrued as tied to neuronal spiking or HFO's based on prior studies [32** $\left.33^{\star \star}\right]$, when in fact, it is a reflection of the PING mechanisms underlying the slower oscillation in the dipole signal. 


\section{Implications for next generation studies of rhythms}

The reviewed results suggest several new directions to pursue in our quest to discover the mechanism and meaning of brain rhythms. First, the transient and stochastic nature of some rhythms (Figure 1) is reminiscent of a stochastic point process, albeit on a slower time scale than cell spiking. Thus, methods to study neural dynamics with point process techniques, typically applied to the study of spiking interactions, could be adapted to the study of rhythms. Several techniques have been developed to study coordination of spiking activity that could be applied to understand the coordination of transient rhythmic events in different brain regions [5961].

Second, to date, attempts to uncover the role of rhythms in function with direct causal manipulations (e.g. optogenetics, tACS, rTMS) typically apply repeated cycles of modulation at the frequency of interest $[14,15,58]$. Given that rhythms can be event-like and intermittent in time, causal manipulations may be more effective and insightful if they are modified to match the temporal characteristics of the "rhythm" they aim to entrain, and the actually underlying mechanism.

Third, new metrics to define a "rhythmic" process should be constructed based on the potentially non-continuous nature of the rhythm. In this vein, Fransen et al., 2015 have proposed a new method to define rhythmicity based on repeatability of activity in adjacent time windows, termed lagged coherence. They applied this measure to distinguish separable alpha and beta components of the somatosensory mu rhythm $\left[62^{\star}, 63\right]$.

Fourth, the fact that oscillatory signals with vastly different waveform features can create peaks in the power spectrum at identical frequencies (Figure 2) implies that the definition of "phase" of an oscillation, and any techniques that depends on "phase" calculations (e.g. spike - field coherence, phase dependent closed-loop manipulations), should be carefully constructed. Rather than narrow band passing and de-convolving the signal into sinusoidal components from which phase is defined, as is standard practice, template matching of signal waveform features can provide a more accurate means to define phase when the raw signal is non-sinusoidal (e.g. matching pursuit algorithms $\left.\left[56^{\star}\right]\right)$. Along these lines, if more studies quantify waveform features 
associated with rhythms in a specific frequency band, we may be able to derive a dictionary of filters that can be used to interpret the mechanisms of a rhythm. This process would be akin to applying algorithms like sort spiking from extracellular field recordings [64], albeit for rhythms the "ground truth" waveforms would likely differ across recording scales.

Fifth, methods to detect asymmetric waveforms including sharp edges (Figure 3 ) should be

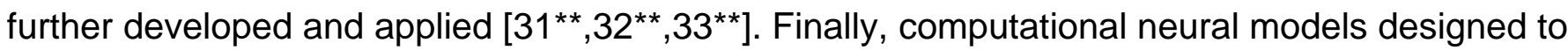
delineate the cellular and circuit level mechanisms creating rhythms should aim to accurately reflect the unfiltered waveform features (Figure 2), and as important, the biophysics of the signals they simulate.

\section{Conclusions}

Defining a functional role for brain rhythms relies on accurate knowledge of the temporal features of the signals from which they emerge. We reviewed several studies that showed rhythms are not always "rhythmic" in the sense of multiple repeated cycles of activity, and that vastly different waveforms can create similar peaks in spectral power. The implication of these facts on the interpretation of the mechanisms and thus the meaning of rhythms is profound. They emphasize the necessity for the field to take a step back from presenting and interpreting rhythms after many levels of filtering and averaging. By developing new methods that take features of raw signals into account, new theories on the role of rhythms in function, and new strategies for manipulating rhythms to improve function, can emerge.

Acknowledgements Work and writing were supported by the National Institutes of Mental Health (R01MH106174) and the National Science Foundation (1131850). Thanks to Manual Gomez-Ramirez, Robert Law, Shane Lee, Chris Moore, and Hyeyoung Shin for helpful comments. 


\section{Figure Legends}

Figure 1 Transient bouts of high power on individual trials appear continuous in the average spectrogram. Top: Ten example trials of time-frequency spectrogram data showing brief bouts of high power (A) and corresponding waveforms (B); axis as in the averages over 100 trials (bottom). Red boxes in $\mathbf{B}$ highlight unfiltered time-domain transients during high power beta events. Data represents MEG activity source localized to SI, during spontaneous prestimulus time periods, as in $\left[26^{\star}, 27^{\star \star}\right]$; power units are in $(\mathrm{Am})^{2}$. Averaging positive-only signals in the frequency domain results in continuous high power in the alpha and beta bands in the averaged spectrogram (bottom A). Averaging positive and negative signal in the time domain results in canceling such that the spectrogram of the averaged waveform has low power in the alpha and beta bands (bottom B: note power scale is $10 x$ smaller than in $\mathbf{A}$ ) [39**].

Figure 2 Vastly different waveforms create transient events with the same peak frequency and approximate duration. Spectrograms (i) and corresponding waveforms (ii) from 3 cycles of a $22 \mathrm{~Hz}$ sine wave (A), an inverted Ricker wavelet (B) and a single brief deflection (C). The peak frequency $(22 \mathrm{~Hz})$ and approximate duration of each transient are the same despite vastly different waveforms likely created by different underlying circuit mechanisms, depicted schematically (see text for details). Each spectrogram was calculated with the same 7-cycle Morlet wavelet convolutions as in [26*].

\section{Figure 3 Waveform features can create spurious high gamma frequency activity and} phase-amplitude coupling with lower frequency rhythms. A A $22 \mathrm{~Hz}$ sawtooth waveform and corresponding spectrogram shows spurious peaks in gamma power at $44 \mathrm{~Hz}$ and $>60 \mathrm{~Hz}$ due to sharp transitions in the waveform, see also [31**]. B. (i) Simulated current dipole signal and corresponding spectrogram created via PING type gamma generating mechanisms (ii top: spike rasters and histogram; red inhibitory; black excitatory), as in [30*]. PING mechanisms creating the $\sim 50 \mathrm{~Hz}$ low gamma oscillation also induce a single cycle of a high frequency oscillation $(\sim 110 \mathrm{~Hz})$ in the current dipole signal (see red dashed line Bii middle-bottom panel; time window corresponds to red box in $\mathrm{Bi}$ ) creating time-locked periods of low and high gamma activity in the spectrogram (Bii bottom panel, i.e., "cross frequency coupling"). The high frequency gamma activity does not reflect an edge effect or distinct neuronal process. Rather, high-gamma is a reflection of the PING mechanisms underlying the slower oscillation in the dipole signal. 
References and recommended reading.

* of special interest

** of outstanding interest

1. Jones SR, Kerr CE, Wan Q, Pritchett DL, Hämäläinen M, Moore C: Cued Spatial Attention Drives Functionally-Relevant Modulation of The Mu Rhythm in Primary Somatosensory Cortex. Journal of Neuroscience 2010, 30:13760-13765.

2. Sacchet MD, LaPlante RA, Wan Q, Pritchett DL, Lee AK, Hamalainen M, Moore Cl, Kerr CE, Jones SR: Attention drives synchronization of alpha and beta rhythms between right inferior frontal and primary sensory neocortex. J Neurosci 2015, 35:2074-2082.

3. Kopell NJ, Gritton HJ, Whittington MA, Kramer MA: Beyond the connectome: the dynome. Neuron 2014, 83:1319-1328.

4. Buzsaki G: Rythms of the Brain. New York: Oxford University Press; 2006.

5. Wang $\mathrm{XJ}$ : Neurophysiological and computational principles of cortical rhythms in cognition. Physiol Rev 2010, 90:1195-1268.

This comprehensive review detailed experimental and computational modeling studies of cerebral cortical oscillations and their functional implications during various cognitive states and mental disorders. It begins with an overview of single neurons as building blocks for oscillations and transitions to reviewing network mechanisms rhythms. Of particular interest is part A of section $\mathrm{VI}$, which highlights several methodological considerations when studying rhythms. In this section, Wang described a number of issues to consider in the measurement, analysis, and fundamental understanding of the dynamic nature of of brain rhythms.

6. Morillon B, Schroeder CE, Wyart V, Arnal LH: Temporal Prediction in lieu of Periodic Stimulation. J Neurosci 2016, 36:2342-2347.

7. Fiebelkorn IC, Saalmann YB, Kastner S: Rhythmic sampling within and between objects despite sustained attention at a cued location. Curr Biol 2013, 23:2553-2558. 
8. Pritchett $\mathrm{DL}$, Siegle JH, Deister CA, Moore Cl: For things needing your attention: the role of neocortical gamma in sensory perception. Curr Opin Neurobiol 2015, 31:254-263. 9. Schroeder CE, Lakatos P: Low-frequency neuronal oscillations as instruments of sensory selection. Trends Neurosci 2009, 32:9-18.

10. Little $S$, Brown P: The functional role of beta oscillations in Parkinson's disease. Parkinsonism Relat Disord 2014, 20 Suppl 1:S44-48.

11. Khan S, Michmizos K, Tommerdahl M, Ganesan S, Kitzbichler MG, Zetino M, Garel KL, Herbert MR, Hamalainen MS, Kenet T: Somatosensory cortex functional connectivity abnormalities in autism show opposite trends, depending on direction and spatial scale. Brain 2015, 138:1394-1409.

12. Gonzalez-Burgos G, Cho RY, Lewis DA: Alterations in cortical network oscillations and parvalbumin neurons in schizophrenia. Biol Psychiatry 2015, 77:1031-1040.

13. Siegle JH, Pritchett $\mathrm{DL}$, Moore $\mathrm{Cl}$ : Gamma-range synchronization of fast-spiking interneurons can enhance detection of tactile stimuli. Nat Neurosci 2014, 17:13711379.

14. Frohlich F: Experiments and models of cortical oscillations as a target for noninvasive brain stimulation. Prog Brain Res 2015, 222:41-73.

15. Thut G, Veniero D, Romei V, Miniussi C, Schyns P, Gross J: Rhythmic TMS causes local entrainment of natural oscillatory signatures. Curr Biol 2011, 21:1176-1185.

16. Ainsworth M, Lee S, Cunningham MO, Traub RD, Kopell NJ, Whittington MA: Rates and rhythms: a synergistic view of frequency and temporal coding in neuronal networks. Neuron 2012, 75:572-583.

17. Lisman JE, Jensen O: The theta-gamma neural code. Neuron 2013, 77:1002-1016.

18. Engel AK, Fries P, Singer W: Dynamic predictions: oscillations and synchrony in topdown processing. Nat Rev Neurosci 2001, 2:704-716.

19. Bastos AM, Vezoli J, Fries P: Communication through coherence with inter-areal delays. Curr Opin Neurobiol 2015, 31:173-180.

20. Harris KD, Csicsvari J, Hirase H, Dragoi G, Buzsaki G: Organization of cell assemblies in the hippocampus. Nature 2003, 424:552-556.

21. Ray S, Maunsell JH: Differences in gamma frequencies across visual cortex restrict their possible use in computation. Neuron 2010, 67:885-896.

22. Ray S, Maunsell JH: Do gamma oscillations play a role in cerebral cortex? Trends Cogn Sci 2015, 19:78-85. 
23. Shadlen MN, Newsome WT: Noise, neural codes and cortical organization. Curr Opin Neurobiol 1994, 4:569-579.

24. Shadlen MN, Newsome WT: The variable discharge of cortical neurons: implications for connectivity, computation, and information coding. J Neurosci 1998, 18:38703896.

25. Merker B: Cortical gamma oscillations: the functional key is activation, not cognition. Neurosci Biobehav Rev 2013, 37:401-417.

26. Jones SR, Pritchett DL, Sikora MA, Stufflebeam SM, Hamalainen M, Moore Cl: Quantitative analysis and biophysically realistic neural modeling of the MEG mu rhythm: rhythmogenesis and modulation of sensory-evoked responses. $J$ Neurophysiol 2009, 102:3554-3572.

* This study combined human MEG imaging and biophysically principled computational neural modeling of current dipole signals to study the cellular and network level mechanisms underlying the somatosensory mu rhythm. Additionally, they applied this integrated approach to interpret the impact of mu on the filtering of sensory evoked responses. The study concluded that the spontaneous rhythm, which consisted of brief and intermittent alpha $(7-14 \mathrm{~Hz})$ and beta $(15-29 \mathrm{~Hz})$ components in unaveraged data, emerged from the integration of two stochastic $10 \mathrm{~Hz}$ excitatory synaptic inputs targetting pyramidal neuron (PN) dendrites in supragranular and infragranular layers. The synaptic inputs drove current flow up and down within the PN dendrites to create the macroscale current dipole rhythm. Alpha or beta expression depended on the relative strength and delay between the two drives on any cycle of the input. Alpha emerged when the inputs were a-synchronous, and beta emerged when the inputs were nearly synchronous and the supragranular drive was sufficiently strong. See also Zielger et al. Neuroimage 2010 for a study of the mechanisms underlying changes in mu and sensory evoked responses with healthy aging.

27. Sherman M, Lee S, Haegens S, Law R, Thorn C, Hamalainen M, Moore Cl, Jones SR: Neural Mechanisms of Transient Neocortical Beta Rhythms: Converging Evidence from Computational Modeling, Humans, Monkeys, and Mice. PNAS In Press. 
This study built from Jones et al. J. Neurophys. 2009 and combined human MEG, computational modeling, and laminar LFP recordings in anesthetized mice and awake monkeys to propose a novel mechanism for transient neocortical beta rhythms (15-29 $\mathrm{Hz}$ ). The study showed that high-power beta events from somatosensory and frontal cortex typically lasted $<150 \mathrm{~ms}$ and had a stereotypical non-sinusoidal waveform shape. They concluded that individual beta events emerged from the integration of a broad burst of excitatory synaptic drive to apical dendrites of pyramidal neurons (PNs) that was disrupted by a simultaneous strong burst of excitatory synaptic drive to the distal dendrites, which lasted one beta period. The dominant feature determining the beta event frequency and power was the strong beta period distal drive. Additional features of the data could be reproduced when the two inputs were simulated at $10 \mathrm{~Hz}$, as in Jones et al. J. Neurophys. 2009. Consistency between methods suggested this beta mechanisms was conserved across areas, species and recording modalities.

\section{Feingold J, Gibson DJ, DePasquale B, Graybiel AM: Bursts of beta oscillation} differentiate postperformance activity in the striatum and motor cortex of monkeys performing movement tasks. Proc Natl Acad Sci U S A 2015, 112:13687-13692.

This study analyzed the distributions of duration and power of beta-band $(13-30 \mathrm{~Hz})$ activity recorded in LFP signals from the striatum and motor-premotor cortex of macaque monkeys performing a reaching task. Using a single trial analysis, they showed that beta bursts typically lasted $90-115 \mathrm{~ms}$, and that extended periods of beta band synchronization reflected a modulation in the density of these short bursts. Burst propabilities were region and task-time specific such that in motor cortex they peak following the last movement, while in striatum they peaked after reward and continued through the postperformance period.

29. Lundqvist M, Rose J, Herman P, Brincat SL, Buschman TJ, Miller EK: Gamma and Beta Bursts Underlie Working Memory. Neuron 2016, 90:152-164.

** This study built from a prior computational modeling study (Lundqvist et al. J. Cogn. Neurosci. 2011) that described attractor dynamics underlying theta and gammma power increases and alpha/beta power decreases with memory load. Lundqvist and colleagues 
(2016) tested the prior model predictions with LFP recording from the prefrontal cortex (PFC) of monkeys performing a working memory task. In agreement with the model, using a trial-by-trial analysis they found brief burst of gamma-band activity $(45-100 \mathrm{~Hz})$ accompanied encoding and re-activation of sensory information in recording sites associated with spiking reflecting to be remembered items. Neuronal activity reflecting encoding/decoding correlated with changes in gamma burst rate. Additionally, they showed gamma - alpha $(8-10 \mathrm{~Hz})$ coupling was not related to low frequency periodicity in the gamma activity but rather consistency in the duration of the gamma bursts during the task. Beta band oscillations $(20-35 \mathrm{~Hz})$ were also brief and transient in their data and was interpreted as reflecting a default state because it was interrupted by encoding and decoding. The authors concluded that working memory is not associated with sustained activity but rather discrete oscillatory dynamics and spiking.

30. Lee $S$, Jones SR: Distinguishing mechanisms of gamma frequency oscillations in human current source signals using a computational model of a laminar neocortical network. Front Hum Neurosci 2013, 7:869.

* This study employed a computational model designed to simulated macroscopic scale current dipole (CD) signals underlying human MEG/EEG to investigate features of the signal that could distinquish between alternative neocortical gamma $(30-150 \mathrm{~Hz})$ mechanisms. They quantified features of $C D$ signals simulated with two different gamma generating mechanisms: pyramidal-interneuron gamma (PING) induced by the spiking interactions of excitatory and inhibitory neurons (Whittington et al., Int J Psychophysiol 2000), and subthreshold driven gamma imposed by sub-cortical circuits. They concluded that PING mechanisms could be distinquished by sharp deflections in the signal resulting from strong somatic inhibition onto pyramidal neurons. They also showed that the PING mechanisms created single cycles of high frequency gamma activity $(100-150 \mathrm{~Hz})$ that did not represent a distinct underlying mechanism, such as spiking or a separate high frequency rhythmic process.

31. Kramer MA, Tort AB, Kopell NJ: Sharp edge artifacts and spurious coupling in EEG frequency comodulation measures. J Neurosci Methods 2008, 170:352-357. 
This methods paper used simulated data to show how abrupt changes in voltage signals can produce spurious high and low frequency coupling. They presented several examples that succinctly proved data consisting of sharp edges can produce spurious frequency co-modulation. Of particular interest, they suggested four techniques to detect sharp edges that might lead to misinterpretation of cross-frequency coupling: (1) visual inspection of unfiltered data, (2) event related averaging of the unfiltered data triggered by the peaks of successive high frequency waves, (3) searching for harmonic peaks of the dominant low frequency oscillation in power spectrum, and (4) computing bicoherence of the raw data. They further suggested that more sophisticated coupling measures of synchronization or causality might help eliminate spurious detection of coupling while maintaining true phase-amplitude correlations.

\section{Ray S, Maunsell JH: Different origins of gamma rhythm and high-gamma activity in} macaque visual cortex. PLoS Biol 2011, 9:e1000610.

** This study recorded LFP and spiking activity from primary visual cortex of awake monkeys during presentation of a series of gratings of different sizes and orientations that increase gamma power but decreased firing rate in the receptive field recording site. They found that low $(30-80 \mathrm{~Hz})$ and high gamma $(>80 \mathrm{~Hz})$ power became anticorrelated reflective of distinct origins. Only the high gamma activity remained strongly correlated with spiking activity under varied stimulus conditions. Using a Matching Pursuit technique (Mallet and Zhang, IEEE Trans Signal Proc 1993) they reported that action potentials were associated with sharp transients in the LFP with broadband power representing "spike bleed-through". They concluded high-gamma activity was a reliable electrophysiological index of neuronal firing near the electrode.

33. Scheffer-Teixeira R, Belchior $H$, Leao RN, Ribeiro $S$, Tort AB: On high-frequency field oscillations ( $>100 \mathrm{~Hz})$ and the spectral leakage of spiking activity. J Neurosci 2013 , 33:1535-1539.

This study used multisite recordings in the hippocampus of freely moving rats and a cross frequency coupling (CFC) approach (Tort et al. J Neurophysiol. 2010) to show that highfrequency LFP oscillations $(>100 \mathrm{~Hz}$ ) can either represent spectral leakage of spiking 
activity (e.g. Ray and Maunsell PLoS Biol 2011) or a genuine rhythm. In either case, the high frequency activity was coupled to the phase of a slower theta frequency oscillation. Of particular interest, they suggested 5 specific features that could clearly distinguish spike-leaked high frequency oscillations (SLHFO) from true high frequency oscillations (HFOs) in this data.

34. Berger H: On the electroencephalogram of man. Electroencephalogr Clin Neurophysiol 1969:Suppl 28:37+.

35. Sejnowski TJ, Destexhe A: Why do we sleep? Brain Res 2000, 886:208-223.

36. O'Keefe J, Recce ML: Phase relationship between hippocampal place units and the EEG theta rhythm. Hippocampus 1993, 3:317-330.

37. Freyer F, Aquino K, Robinson PA, Ritter P, Breakspear M: Bistability and non-Gaussian fluctuations in spontaneous cortical activity. J Neurosci 2009, 29:8512-8524.

38. Leventhal DK, Gage GJ, Schmidt R, Pettibone JR, Case AC, Berke JD: Basal ganglia beta oscillations accompany cue utilization. Neuron 2012, 73:523-536.

39. Tallon-Baudry C, Bertrand $O$ : Oscillatory gamma activity in humans and its role in object representation. Trends Cogn Sci 1999, 3:151-162.

** This earlier review detailed analysis methods that distinquished time-locked "evoked" oscillations versus non-time locked "induced" oscillation. In Box 2 of this paper, they used simulated EEG data to describe how "evoked" oscillations could be visuzalized by first computing evoked potentials by averaging across stimulus locked trials, and second applying a Morlet wavelet transform to average data. In contrast, "induced" oscillations could be vizualized by applying the wavelet transform to individual trial data, and then averaging the spectral power across trials. Averaging in the spectral domain results in the accumulation of non-time locked transient oscillations. They reviewed results applying this technique that suggested induced gmma band activity played a role in the construction of visual object represetation. Of particular relevance, they described the transient nature of gamma rhythms $(25-45 \mathrm{~Hz})$ in EEG signals and detailed a prior study that showed transient gamma activity typically lasted $100-150 \mathrm{~ms}$, with a jitter across trials of up to 40ms (Tallon-Baudrey et al. J. Neurosci. 1997). 
40. Tallon-Baudry C, Bertrand O, Delpuech C, Permier J: Oscillatory gamma-band $(\mathbf{3 0 - 7 0 ~} \mathrm{Hz})$ activity induced by a visual search task in humans. J Neurosci 1997, 17:722-734.

41. Cohen MX, Donner TH: Midfrontal conflict-related theta-band power reflects neural oscillations that predict behavior. J Neurophysiol 2013, 110:2752-2763.

* $\quad$ This study addressed the debate of whether conflict related $(4-8 \mathrm{~Hz})$ theta-band power in midfrontal cortex EEG signals reflects non-phased neural oscillations or phase-locked non-oscillatory responses (i.e., event-related potentials). They described methods to remove the phase-locked component and concluded that most of the conflict-related EEG signal reflects a modulation of ongoing theta-band oscillations that are not phase-locked to the stimulus or to the response.

42. Delorme A, Makeig S: EEGLAB: an open source toolbox for analysis of single-trial EEG dynamics including independent component analysis. J Neurosci Methods 2004, 134:9-21.

43. Lachaux JP, Rodriguez E, Martinerie J, Varela FJ: Measuring phase synchrony in brain signals. Hum Brain Mapp 1999, 8:194-208.

44. Yeung N, Bogacz R, Holroyd CB, Nieuwenhuis S, Cohen JD: Theta phase resetting and the error-related negativity. Psychophysiology 2007, 44:39-49.

45. Trujillo LT, Allen JJ: Theta EEG dynamics of the error-related negativity. Clin Neurophysiol 2007, 118:645-668.

46. Mazaheri A, Jensen $O$ : Asymmetric amplitude modulations of brain oscillations generate slow evoked responses. J Neurosci 2008, 28:7781-7787.

47. Whittington MA, Traub RD, Kopell N, Ermentrout B, Buhl EH: Inhibition-based rhythms: experimental and mathematical observations on network dynamics. Int $\mathrm{J}$ Psychophysiol 2000, 38:315-336.

** This foundational review described slice experiments with pharmacological manipulations, and computational modeling studies, that established how rhythms in the $12-80 \mathrm{~Hz}$ range could be generated by the spiking interactions of cells. They showed that the period of the gamma rhythm $(30-80 \mathrm{~Hz})$ was set by the time constant of GABA $A$ inhibition, and coined the terms "ING" (interneuron network gamma oscillations) and "PING" (pyramidal-interneuron network gamma oscillations). In both ING and PING, 
excitable cells in the network fired action potentials only when synaptic inhibition had sufficiently decayed resulting in a gamma frequency LFP oscillation. Cells in the network could spike either synchronously or sparsely depending on network configurations. They also described how slower beta frequency rhythms $(20-30 \mathrm{~Hz})$ could emerge from similar mechanisms when the effects of inhibition were effectively prolonged (lengthening the period of the oscillation) either by slower decay of the inhibitory synapses, or slow intrinsic properties of the excitatory cells (e.g. AHP currents). Several follow up papers further detailed synaptic and intrinsic mechanisms contributing to beta frequency oscillations in spiking networks (e.g. Roopun et al., PNAS 2006; Kramer et al. PLoS Comput. Biol. 2008).

48. Roopun AK, Middleton SJ, Cunningham MO, LeBeau FE, Bibbig A, Whittington MA, Traub $\mathrm{RD}$ : A beta2-frequency $(20-30 \mathrm{~Hz})$ oscillation in nonsynaptic networks of somatosensory cortex. Proc Natl Acad Sci U S A 2006, 103:15646-15650.

49. Kramer MA, Roopun AK, Carracedo LM, Traub RD, Whittington MA, Kopell NJ: Rhythm generation through period concatenation in rat somatosensory cortex. PLOS Comput Biol 2008, 4:e1000169.

50. Jones SR, Pritchett DL, Stufflebeam SM, Hamalainen M, Moore Cl: Neural Correlates of

Tactile Detection: A Combined MEG and Biophysically Based Computational Modeling Study. Journal of Neuroscience 2007, 27:10751-10764.

51. Jones SR: Local Field Potential, Relationship to Electroencephalogram (EEG) and Magnetoencephalogram (MEG). In Encylopedia of Computational Neuroscience: SpringerReference. Edited by Jaeger D. JR: Springer-Verlag Berlin Heidelberg; 2014.

* This encyclopedia contribution detailed the mathematical and biophysical connection between LFP and MEG/EEG recordings. Of particular interest, this contribution described how primary current dipole sources commonly estimated from MEG/EEG data could be modeled from the summed post-synaptic intracellular current flow within long and spatially aligned cortical pyramidal neuron dendrites. In contrast, biophysically principled models of LFP signals need account for greater detail in the local network and extracelluar conductivity gradients. 
52. Canolty RT, Edwards E, Dalal SS, Soltani M, Nagarajan SS, Kirsch HE, Berger MS, Barbaro NM, Knight RT: High gamma power is phase-locked to theta oscillations in human neocortex. Science 2006, 313:1626-1628.

53. Colgin LL: Theta-gamma coupling in the entorhinal-hippocampal system. Curr Opin Neurobiol 2015, 31:45-50.

54. Cohen MX: Analyzing Neural Time Series Data: Theory and Practice MIT Press; 2014.

55. Kass RD, Eden U, Brown EN: Analysis of Neural Data: Springer; 2014.

56. Mallat $S$, Zhang Z: Matching pursuit with time-frequency dictionaries. . IEEE Trans Signal Proc 1993, 41:3397-3415.

* This study described a method that iteratively decomposes a signal into a linear expansion of waveforms selected from a large overcomplete dictionary, with waveforms chosen to best match the local signal structures. If a dictionary of Gabor functions is applied, a matching pursuit algorithm defines an adaptive time-frequency transform.

57. Tiesinga $P$, Sejnowski TJ: Cortical enlightenment: are attentional gamma oscillations driven by ING or PING? Neuron 2009, 63:727-732.

58. Cardin JA, Carlen M, Meletis K, Knoblich U, Zhang F, Deisseroth K, Tsai LH, Moore Cl: Driving fast-spiking cells induces gamma rhythm and controls sensory responses. Nature 2009, 459:663-667.

59. Zandvakili A, Kohn A: Coordinated Neuronal Activity Enhances Corticocortical Communication. Neuron 2015, 87:827-839.

60. Czanner G, Eden UT, Wirth S, Yanike M, Suzuki WA, Brown EN: Analysis of between-trial and within-trial neural spiking dynamics. J Neurophysiol 2008, 99:2672-2693.

61. Amarasingham A, Harrison MT, Hatsopoulos NG, Geman S: Conditional modeling and the jitter method of spike resampling. J Neurophysiol 2012, 107:517-531.

62. Fransen AM, van Ede F, Maris E: Identifying neuronal oscillations using rhythmicity. Neuroimage 2015, 118:256-267.

* $\quad$ This study presented a method to quantify rhythmicity, termed "lagged coherence", which calculates the extent to which future phases of an oscillation can be predited from the present one. They applied this method to identify alpha and beta components in ongoing MEG signals from sensorimotor cortex and showed that they could be differentiated from 
posterior activity. They concluded that phase-lagged rhythmicity of neuronal activity is better suited to identify neural oscillations than power. In a follow up study (Fransen et al. J. Neurophysiol. 2016), they applied this measure to distinguish alpha and beta band activity in rat sensorimotor cortex and concluded beta activity emerged independently of alpha.

63. Fransen AM, Dimitriadis G, van Ede F, Maris E: Distinct alpha and beta band rhythms over rat somatosensory cortex with similar properties as in humans. J Neurophysiol 2016:jn 0050702015.

64. Quian Quiroga R: What is the real shape of extracellular spikes? J. Neurosci. Methods 2009, 117:194-198. 
A
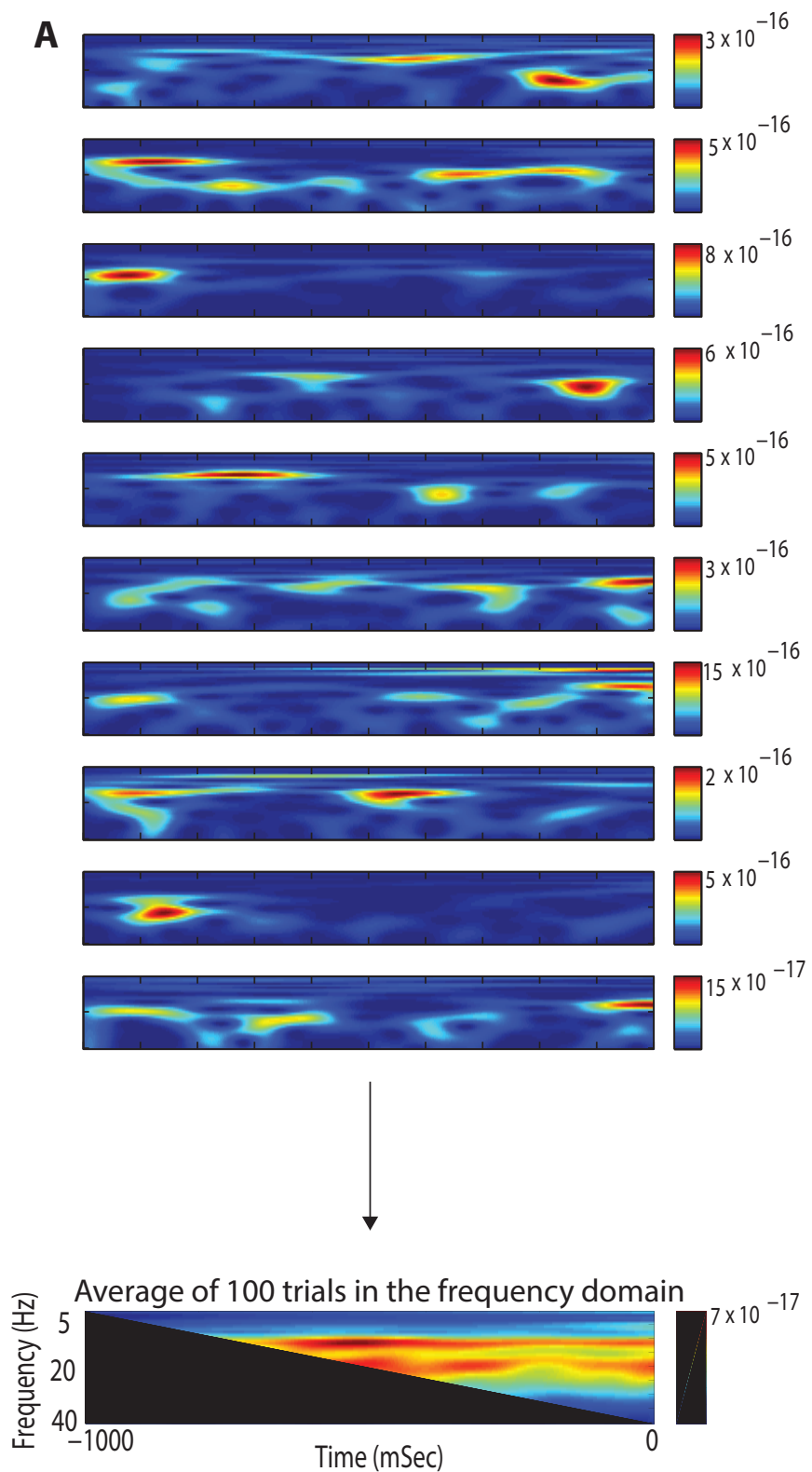

B

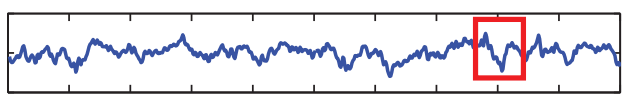

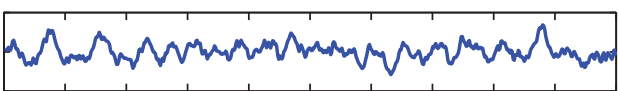

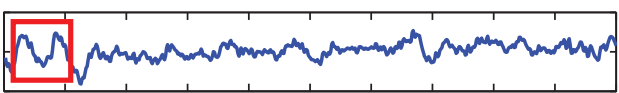

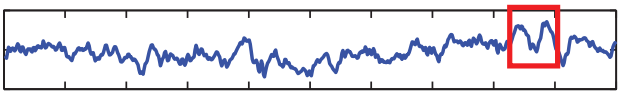

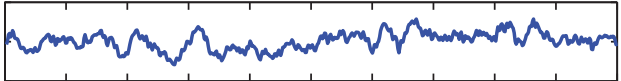

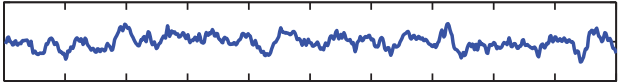

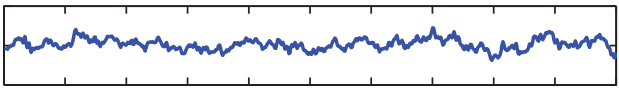

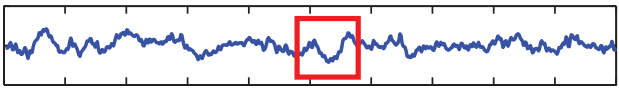

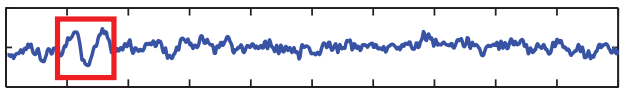
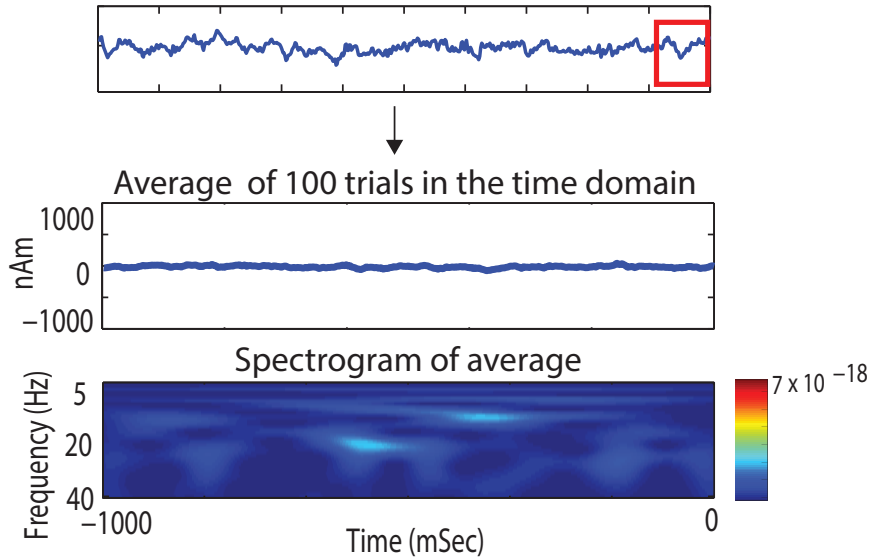
Figure 2
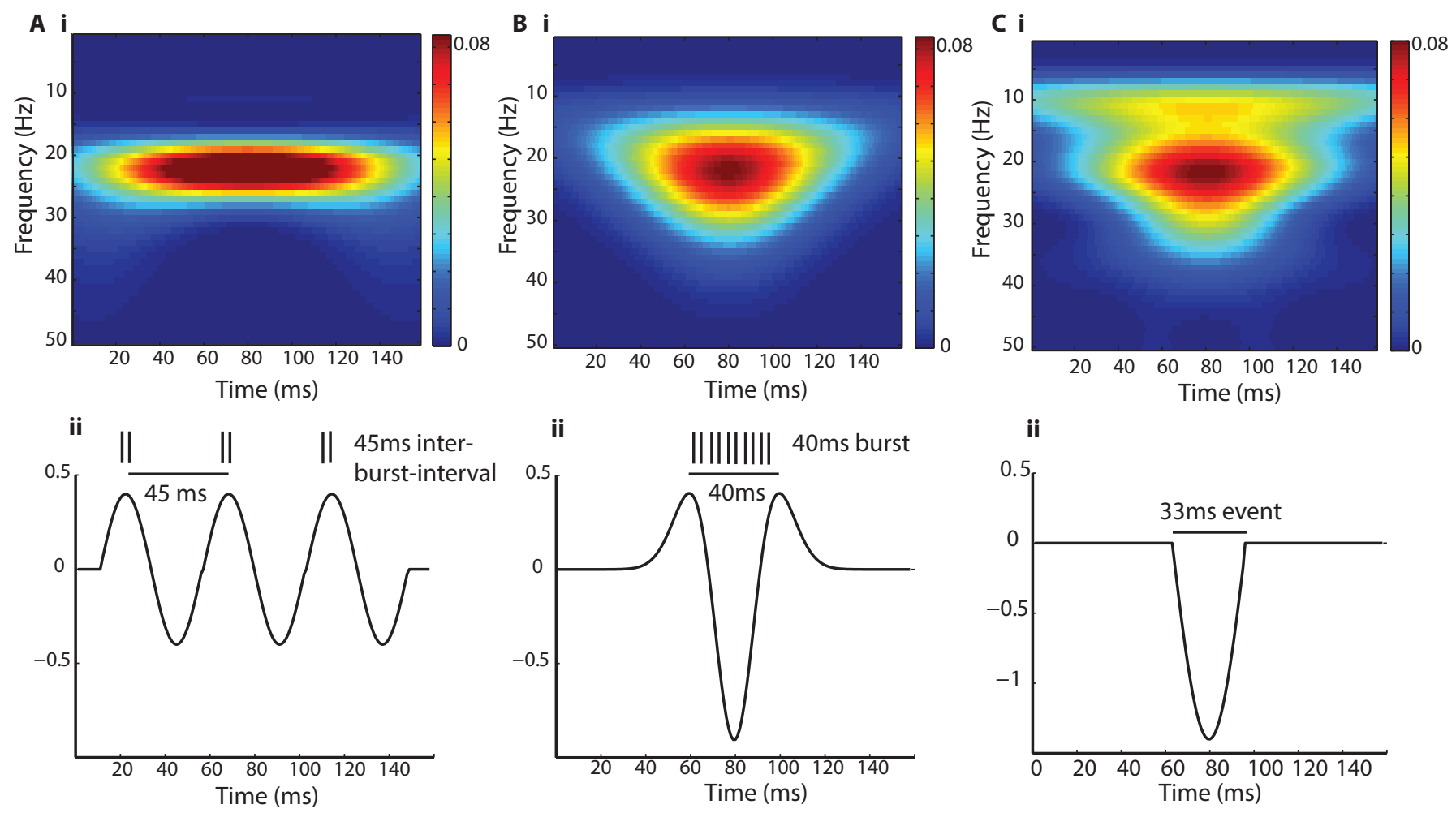
A
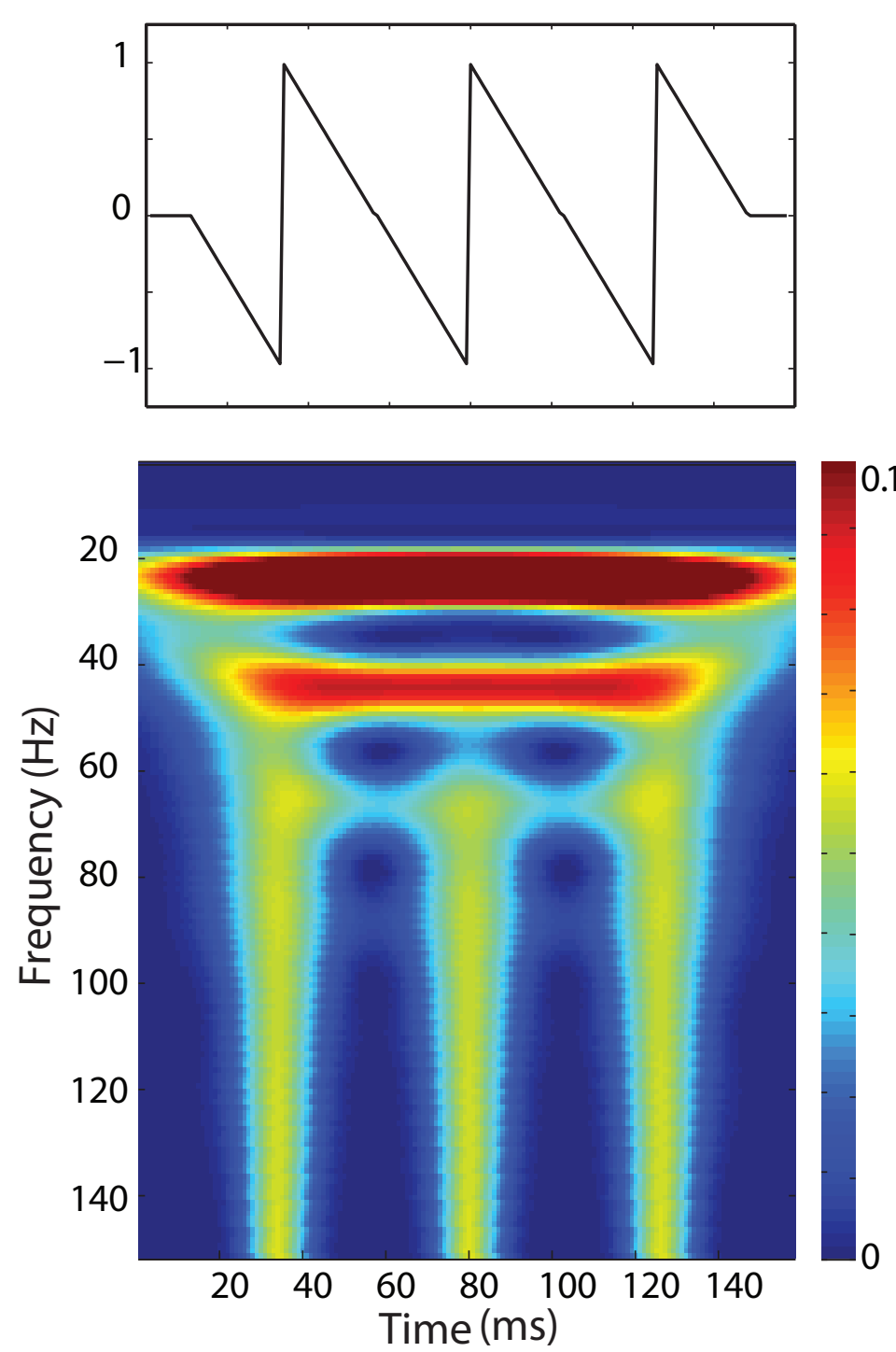

B i
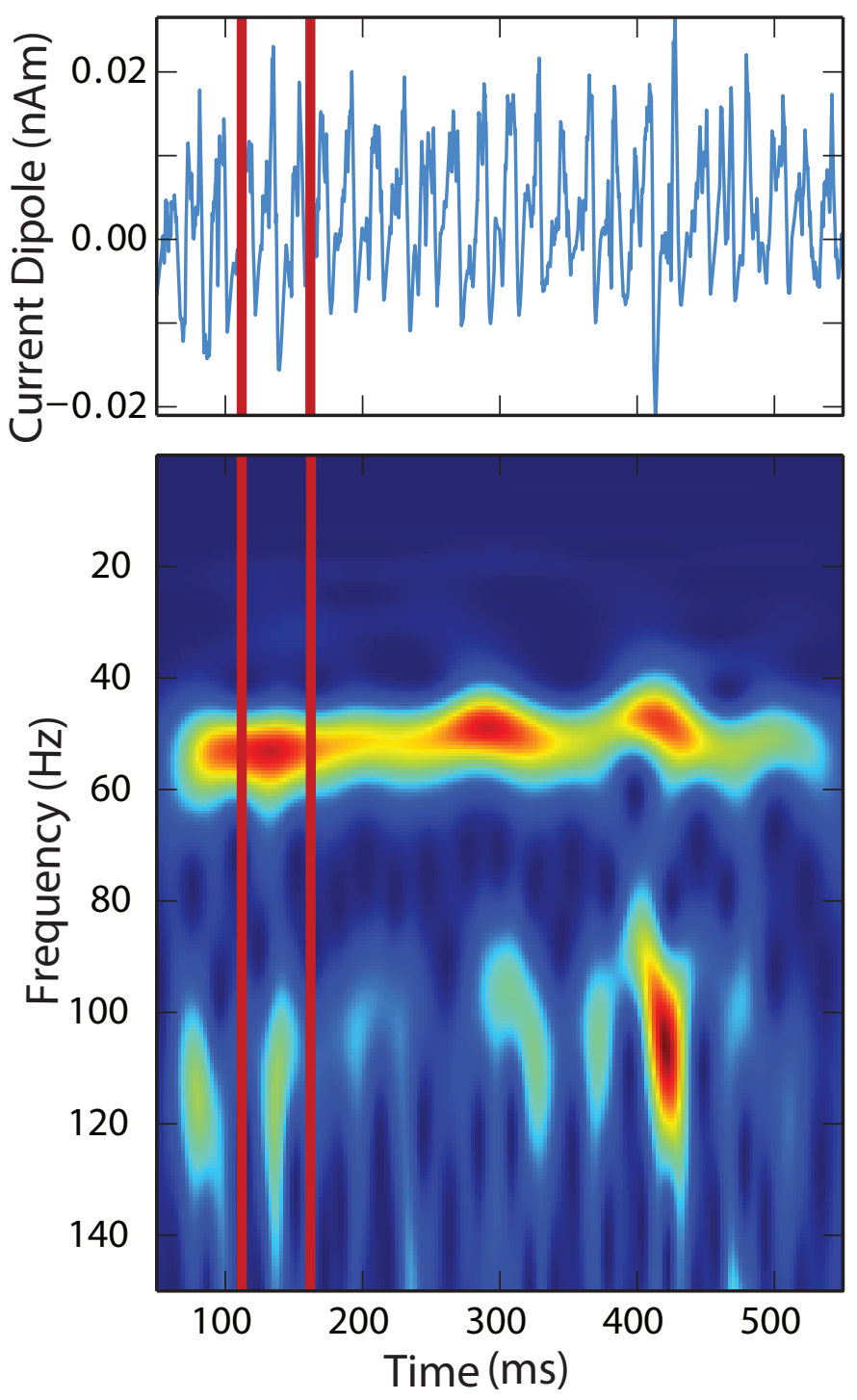

ii
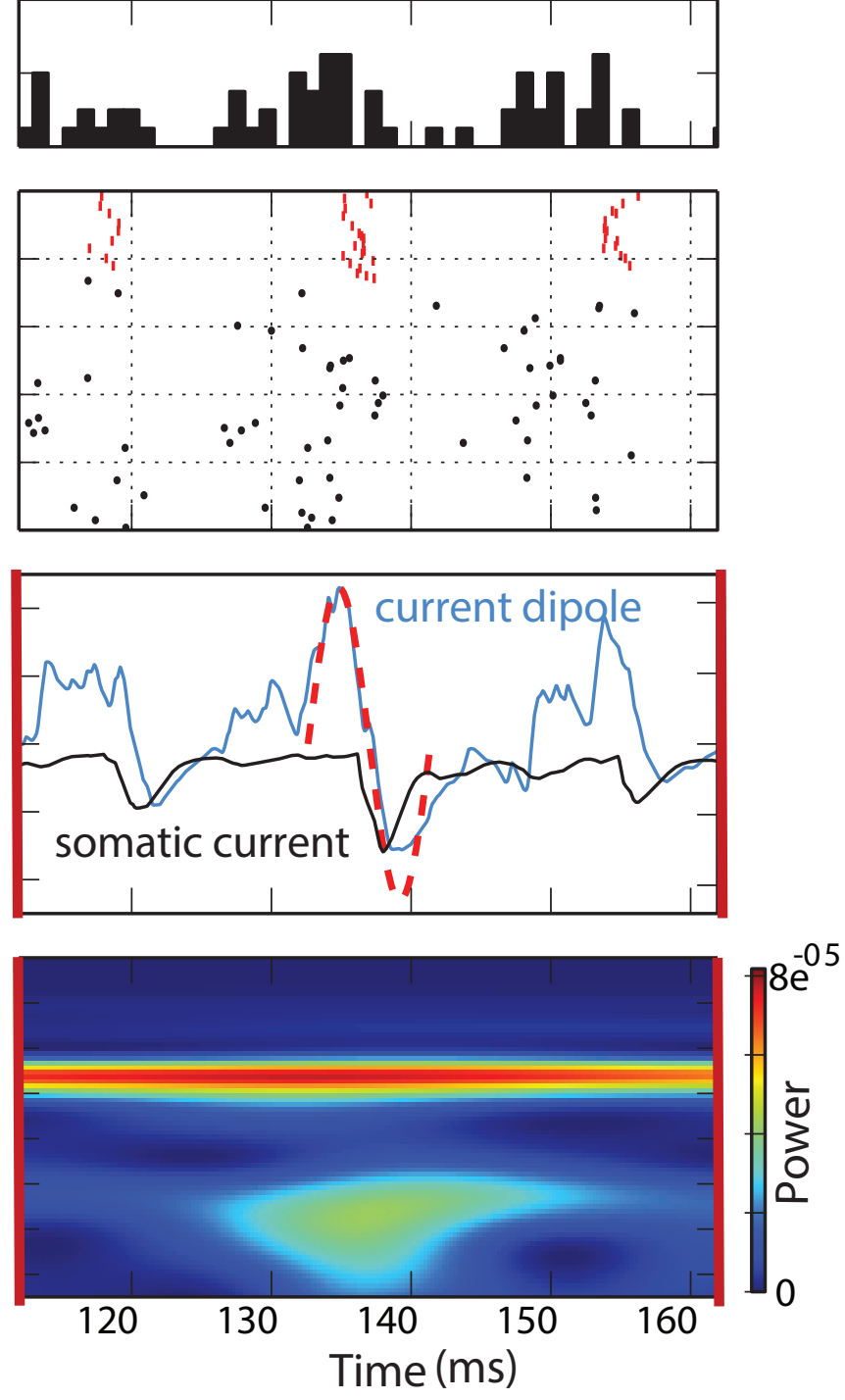